\title{
Diagnostic Algorithm in Hirschsprung's Disease: Focus on Immunohistochemistry Markers
}

\author{
PRZEMYSLAW GALAZKA ${ }^{1,2}$, LUKASZ SZYLBERG $^{3}$, MAGDALENA BODNAR $^{3}$, \\ JAN STYCZYNSKI ${ }^{2}$ and ANDRZEJ MARSZALEK ${ }^{3,4}$ \\ ${ }^{1}$ Department of General and Oncological Surgery for Children and Adolescents, \\ Nicolaus Copernicus University in Torun, Ludwik Rydygier Collegium Medicum, Bydgoszcz, Poland; \\ ${ }^{2}$ Department of Pediatric Hematology and Oncology, Nicolaus Copernicus University in Torun, \\ Ludwik Rydygier Collegium Medicum, Bydgoszcz, Poland; \\ ${ }^{3}$ Department of Clinical Pathology, Nicolaus Copernicus University in Torun, \\ Ludwik Rydygier Collegium Medicum, Bydgoszcz, Poland; \\ ${ }^{4}$ Department of Oncologic Pathology and Prophylaxis, \\ Poznan University of Medical Sciences \& Greater Poland Cancer Center, Poznan, Poland
}

\begin{abstract}
Background/Aim: Hirschsprung disease (HD) is caused by the congenital absence of ganglion cells in the distal bowel (aganglionosis). Rectal biopsy is considered important for its diagnosis. The aim of this study was to apply immunohistochemical staining using a minimal set of antibodies and develop an algorithm that will assist in the diagnosis of HD. Patients and Methods: Rectal or colonic biopsies were performed in patients with $H D(n=26)$ or patients treated for other bowel diseases $(n=34)$. Immunohistochemical staining was performed for MAP1b, peripherin, S-100, calretinin, NSE, bcl-2 and CD56 proteins. Results: Staining for CD56, S-100, peripherin and calretinin facilitated the identification of ganglion cells. The use of CD56 and S-100 antibodies together resulted in the highest rate of ganglion cell staining intensity (94\%). Conclusion: We propose a practical diagnostic algorithm with the application of CD56 and S-100 antibodies that can be used in clinical practice in children suspected of Hirschsprung's disease.
\end{abstract}

Hirschsprung disease (HD) is a congenital, genetically-based functional obstruction due to the congenital absence of

This article is freely accessible online.

Correspondence to: Przemysław Gałązka MD, Ph.D., Department of General and Oncological Surgery for Children and Adolescents, Jurasz University Hospital, Nicolaus Copernicus University Toruń, Collegium Medicum, ul. Sklodowskiej-Curie 9, 85-094 Bydgoszcz, Poland. Tel: +48 525854015, Fax: +48 525854093, e-mail: galazkaprzemek@hotmail.com

Key Words: Immunohistochemistry, CD56, S-100, calretinin, peripherin, ganglion cells, Hirschsprung's disease. ganglion cells in the distal bowel (aganglionosis) $(1,2)$. The absence of ganglion cells along with an analysis of hypertrophy and hyperplasia of nerves in the nerve plexus of submucosa and muscularis mucosae in rectal biopsy specimens (RB) are regarded as potential hallmarks for its diagnosis (3). Hematoxylin and eosin (HE) staining alone in the assessment of the presence of ganglion cells (GC) is still challenging, especially in newborns with total colonic aganglionosis (TCA) or intestinal neuronal dysplasia (IND) (4). Diagnosis of the disease is mainly based on the identification of the lack of ganglion cells in the pathology sections of the colon and often requires an additional immunohistochemical (IHC) staining, which is very difficult and time consuming and also needs several serially cut sections $(5,6)$.

This study was performed in order to ascertain the role of IHC staining with a selected set of antibodies in diagnosing HD and develop a diagnosis algorithm based on the selection of a minimal set of antibodies sufficient for its diagnosis.

\section{Patients and Methods}

Patients. A total of 60 children were enrolled in the study, including 26 with clinical symptoms of aganglionosis (HD, Group 1) and 34 with other diseases, such as meconium ileus, necrotizing enterocolitis, chronic constipation or sigmoid volvulus (non-HD, Group 2). All patients with aganglionosis (Group 1) and 24/34 with non-HD (Group 2) were male. Median age was the same in both groups: 9 months (range $=6-30$ months) and $73 \%$ and $76 \%$ were under 1 year, respectively. In all cases, rectal or colonic biopsies were performed and the specimens were archived in formalin-fixed paraffin-embedded tissue sections, followed by HE and IHC staining.

Immunohistochemistry (IHC). The IHC staining was performed using a set of antibodies against: MAP1b neuronal marker (Abcam, Cambridge, UK), peripherin (Novocastra, Newcastle, UK), S-100 

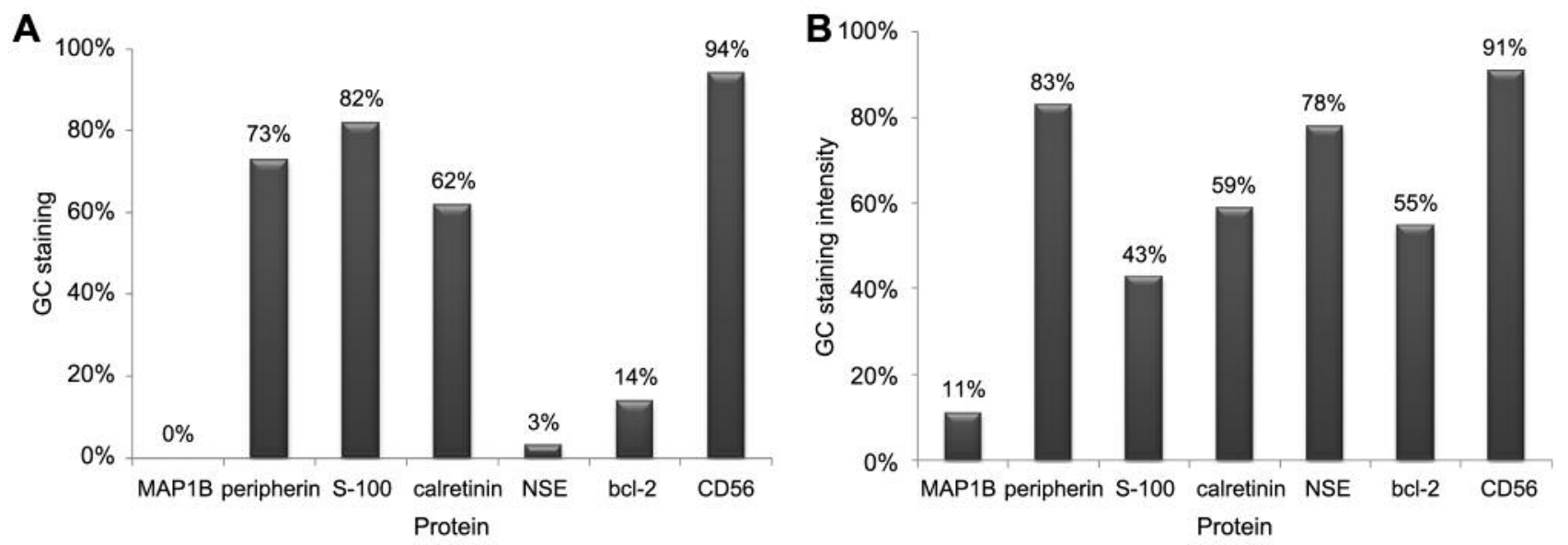

Figure 1. Immunohistochemical staining: (A) ganglion cells distinction index; (B) ganglion cells staining intensity rate.

(DAKO, Glostrup, Denmark), calretinin (DAKO), NSE (DAKO), bcl2 (DAKO) and CD56 (DAKO). To determine the appropriate antibody dilution to eliminate false-positive results, as well as to reduce background reactions, a series of control reactions were performed before adequate immunohistochemical staining. Positive and negative control reactions were performed in each case. The independent assessment was performed by two experienced pathologists.

In order to identify the best set of antibodies for GC identification in IHC staining, the following four criteria were used: 1. possibility of GC distinction from other neural components; 2 . lack of artifacts; 3 . good intensity of GC staining; 4. the highest fulfillment rate comparing results of different antibodies. These tests were performed on non-HD samples (Group 2).

To assess the possibility of GC distinction from other neural components (first criterion), a GC distinction index was created: GC distinction $=\left[\left(0 \times n_{a}+1 / 2 \times n_{m}+n_{e}\right) /\left(n_{a}+n_{m}+n_{e}\right)\right] \times 100 \%$, where: $n_{a}-$ number of samples where GC were absent; $n_{m}$ - number of samples where GC were moderately easy to detect; $n_{e}-$ number of samples where GC were easy to detect. Assessment of GC staining intensity was semiquantitative using 4 groups of results: no staining or artifacts, weak staining of GC, GC well visible and evidently visible. To assess good intensity of GC staining (third criterion), a GC staining intensity index was created: GC staining intensity $=\left[\left(0 \times n_{0}+1 / 3 \times n_{1}+2 / 3 \times n_{2}+n_{3}\right) /\left(n_{0}+n_{1}+n_{2}+n_{3}\right)\right] \times 100 \%$, where: $\mathrm{n}_{0}$ - number of samples with no staining or artifacts; $\mathrm{n}_{1}-$ number of samples where GC were weakly stained; $n_{2}-$ number of samples where GC were moderately stained; $\mathrm{n}_{3}$ - number of samples where GC were evidently stained. The study was approved by the Institutional Review Board, (Department of General and Oncological Surgery for Children and Adolescents, KB 167/2012).

Statistical analysis. Categorical variables were compared with the chi-square or Fisher exact test, and non-categorical variables were compared with the Mann-Whitney $U$-test.

\section{Results}

Four antibodies clearly facilitated the identification of ganglion cells (first criterion) in the IHC studies: CD56, S-
100 , peripherin and calretinin with a more than $60 \%$ index of GC distinction (Figure 1A).

Analysis of the rate of artifacts (second criterion) revealed that anti-S-100 and anti-bcl-2 antibodies were the most efficient ( $0 \%$ of negative staining). A relatively high rate of negative staining was observed for MAP1B (31\%) and calretinin (18\%), while the best intensity of GC staining (third criterion) was found for CD56 (91\%) and peripherin (83\%) (Figure 1B). The overall sensitivity, specificity, positive, and negative predictive value of antibody staining is shown in Table I.

The fourth criterion was assessed using cluster analysis and the largest incompatibility rate (Table II). Two groups of results were defined: the first cluster included MAP1B, S100 and bcl-2, while the second one included peripherin, CD56, NSE and calretinin. As only four antibodies clearly facilitated the identification of ganglion cells (Figure 1A), available sets of antibodies included: CD56 and S-100, peripherin and S-100, and calretinin and S-100 with summary marker expression indexes: $94 \%, 88 \%$ and $74 \%$, respectively. The identification of ganglion cells was the most efficient for the set of CD56 and S-100, in cases without artifact staining.

In order to verify the value of IHC in patients with aganglionosis, an expanded set of IHC studies was performed on biopsy specimens obtained from patients with Hirschsprung`s disease (Figure 2). The staining with CD56, $\mathrm{S}-100$, peripherin and calretinin demonstrated the presence of GC in 3/26 patients initially qualified as aganglionosis in HE examination. In two patients, the morphology of GC was truly dysplastic, and the third patient was further diagnosed and treated for food allergy. All 23/26 HD cases after surgical treatment were uncomplicated, except for more frequent bowel movements and perianal excoriations for a period of 1-2 months in two patients. 
Galazka et al: Diagnostic Algorithm in Hirschsprung's Disease

Table I. Sensitivity, specificity, positive, and negative predictive value of antibody staining of ganglion cells.

\begin{tabular}{lccccccc}
\hline$\%$ & MAP1B & Peripherin & S100 & Calretinin & NSE & Bcl-2 & CD56 \\
\hline Sensitivity & 26.5 & 91.2 & 97 & 76.4 & 97 & 97 & 91.2 \\
Specificity & 95.6 & 95.6 & 100 & 100 & 95.6 & 100 & 100 \\
PPV & 90 & 96.8 & 100 & 100 & 97 & 100 & 100 \\
NPV & 46.8 & 88 & 95.8 & 74 & 95.6 & 95.8 & 88.4
\end{tabular}

PPV: Positive predictive value; NPV: Negative predictive value.

Table II. Percent incompatibility rate between the ganglion cells staining results in the group with a normal ganglionic bowel.

\begin{tabular}{lccccccc}
\hline Antibody & MAP 1B & Peripherin & S-100 & Calretinin & NSE & Bcl-2 & CD56 \\
\hline MAP 1B & - & 0.85 & 0.79 & 0.82 & 0.97 & 0.85 & 0.94 \\
Peripherin & 0.85 & - & 0.94 & 0.62 & 0.59 & 0.82 & 0.21 \\
S-100 & 0.79 & 0.94 & - & 0.88 & 0.88 & 0.59 & 0.91 \\
Calretinin & 0.82 & 0.62 & 0.88 & - & 0.76 & - & 0.85 \\
NSE & 0.97 & 0.59 & 0.88 & 0.76 & 0.85 & 0.53 \\
Bcl-2 & 0.85 & 0.82 & 0.59 & 0.91 & 0.53 & 0.82 & - \\
CD56 & 0.94 & 0.21 & 0.94 & 0.59 & & 0.82 \\
\hline
\end{tabular}

\section{Discussion}

Nowadays, surgical treatment of Hirschsprung disease is performed at an earlier age and more often in a one-stage manner and therefore, requires very precise preoperative diagnosis (7). HE staining of rectal biopsy specimens is a gold standard for HD and differential diagnosis $(2,8,9)$. Proper assessment of GC presence requires primarily adequate tissue sampling and careful tissue preparation. There are numerous reports on the use of various antigens in IHC studies for better diagnosis of aganglionosis. An ideal immunohistochemical marker would facilitate easy detection of GC through good, intensive staining, as well as easy distinction of GC from other neural components, and a low artifact rate (10).

We showed that selection of a pair of IHC assay reagents facilitates the diagnostic examinations of patients tested for HD. In the meantime, high sensitivity, specificity and efficacy of peripherin and S-100 staining was confirmed in a diagnostic protocol for HD. Ganglion cells were diagnosed in $93 \%$ of samples excluded in the primary examination (11). According to our algorithm, rectal biopsies with ambiguous histopathological presentation that does not allow for confirmation or exclusion of HD were subject for internal verification as assessed by another experienced pathologist, and final diagnosis was pursued by means of the proposed set of IHC assays.

A biopsy for the ganglion cells, and consequently, for neural hypertrophy is regarded to be the basic modality for the diagnosis of HD. S-100 staining was shown to be diagnostic in rectal suction biopsies in the samples that were devoid of ganglion cells. Staining for S-100 coupled with calretinin and PGP9.5 immunostaining performed on suction rectal biopsies was shown to be sensitive and specific for diagnosing HD (12). However, S-100 was found to be positive in ovarian cancer (13), metastases to the small intestine (14) and fibrosarcoma cells (15). The other marker, CD56, was used in evaluating a "transition zone" proximally to the aganglionic segment, with the use of serial colonic wall biopsies and obtained very good results (16). CD56 is known to be useful for definitive histopathological diagnosis of isolated hypoganglionosis. In the aganglionic segment in HD patients, CD56-positive extrinsic nerve fibers and bundles replaced typical ganglion cells that are immunoreactive for $\mathrm{Hu} C / \mathrm{D}$ (17). Other recommended combinations of staining included $\mathrm{AChE}$ and calretinin (6) or peripherin and calretinin (18).

The practical value of the study is related to clinical verification: in three cases classified as $\mathrm{HD}$, the use of the examined panel of immunohistochemical reagents allowed for clear visualization of ganglion cells, and therefore for verification of the primary diagnosis of HD. The histopathological diagnostic algorithm presented in this study provides a tool for improving the sensitivity and specificity of assays used in the analysis of specimens and eventually for a reduction in the number of patients unnecessarily subjected to surgical treatment.

In conclusion, we created a practical diagnostic algorithm with the application of CD56 and S-100 markers that can be 


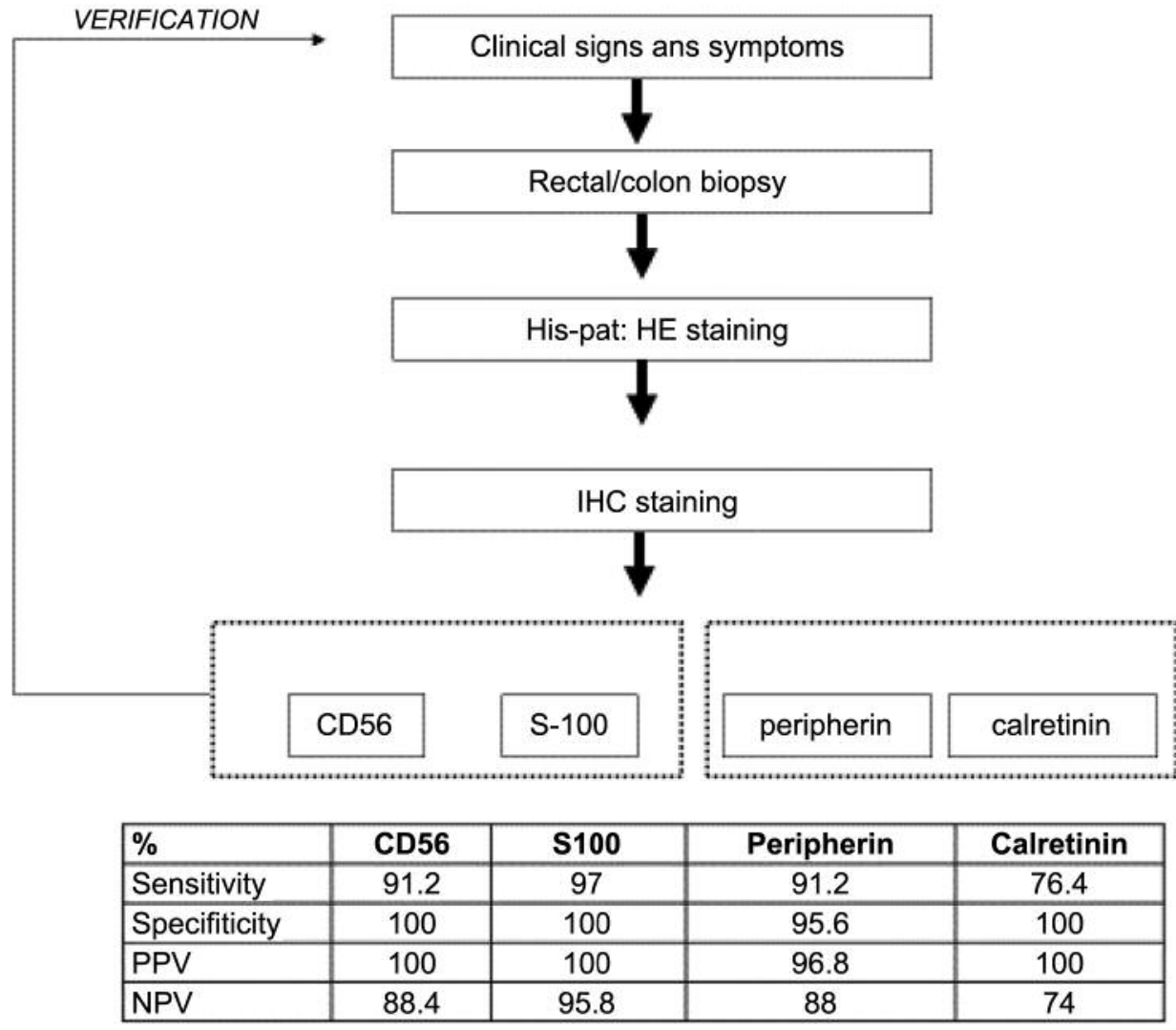

Figure 2. Diagnostic algorithm based on verification of immunohistochemical markers in Hirschsprung's disease.

used in IHC in clinical practice for the diagnosis of HD. Nevertheless, additional studies are needed to confirm the value of calretinin and peripherin in patients diagnosed for aganglionosis.

\section{Conflicts of Interest}

The Authors have no conflicts of interest to disclose in regard to this study.

\section{Authors' Contributions}

PG, AM: concept/design, data analysis/interpretation; LS, MB: data collection, data analysis/interpretation; PG, AM, JS: writing, critical revision and approval of the article.

\section{References}

1 Moore SW: Advances in understanding functional variations in the Hirschsprung disease spectrum (variant Hirschsprung disease). Pediatr Surg Int 33(3): 285-298, 2017. PMID: 27988850. DOI: 10.1007/s00383-016-4038-3

2 Friedmacher F and Puri P: Current practice patterns of rectal suction biopsy in the diagnostic work-up of Hirschsprung's disease: Results from an international survey. Pediatr Surg Int
32(8): 717-722, 2016. PMID: 27350541. DOI: 10.1007/s00383016-3907-0

3 Mukhopadhyay B, Sengupta M, Das C, Mukhopadhyay M, Barman S and Mukhopadhyay B: Immunohistochemistry-based comparative study in detection of Hirschsprung's disease in infants in a tertiary care center. J Lab Physicians 9(2): 76-80, 2017. PMID: 28367019. DOI: 10.4103/0974-2727.199623

4 Qualman SJ, Jaffe R, Bove KE and Monforte-Munoz H: Diagnosis of Hirschsprung disease using the rectal biopsy: Multi-institutional survey. Pediatr Dev Pathol 2(6): 588-596, 1999. PMID: 10508885. DOI: 10.1007/s100249900167

5 Volpe A, Alaggio R, Midrio P, Iaria L and Gamba P: Calretinin, beta-tubulin immunohistochemistry, and submucosal nerve trunks morphology in Hirschsprung disease: Possible applications in clinical practice. J Pediatr Gastroenterol Nutr 57(6): 780-787, 2013. PMID: 23969533. DOI: 10.1097/MPG. $0 \mathrm{~b} 013 \mathrm{e} 3182 \mathrm{a} 934 \mathrm{c} 7$

6 de Arruda Lourencao PL, Takegawa BK, Ortolan EV, Terra SA and Rodrigues MA: A useful panel for the diagnosis of Hirschsprung disease in rectal biopsies: Calretinin immunostaining and acetylcholinesterase histochesmistry. Ann Diagn Pathol 17(4): 352356, 2013. PMID: 23683882. DOI: 10.1016/j.anndiagpath. 2013.04.004

7 De La Torre L and Langer JC: Transanal endorectal pull-through for Hirschsprung disease: Technique, controversies, pearls, pitfalls, and an organized approach to the management of postoperative 
obstructive symptoms. Semin Pediatr Surg 19(2): 96-106, 2010 PMID: 20307846. DOI: 10.1053/j.sempedsurg.2009.11.016

8 Ambartsumyan L, Smith C and Kapur RP: Diagnosis of Hirschsprung disease. Pediatr Dev Pathol 23(1): 8-22, 2020. PMID: 31791203. DOI: 10.1177/1093526619892351

9 Kim J, Ahn S, Kim K, Cho MS, Kim KH, Lee RA and Nam EM: Prognostic significance of survivin expression and combined analysis with cancer stem cell and epithelial-mesenchymal transition-related markers in patients with rectal cancer undergoing preoperative chemoradiotherapy. Anticancer Res 38(12): 6881-6889, 2018. PMID: 30504405. DOI: 10.21873/ anticanres. 13064

10 Kapur RP, Reed RC, Finn LS, Patterson K, Johanson J and Rutledge JC: Calretinin immunohistochemistry versus acetylcholinesterase histochemistry in the evaluation of suction rectal biopsies for Hirschsprung disease. Pediatr Dev Pathol 12(1): 6-15, 2009. PMID: 18442301. DOI: 10.2350/08-020424.1

11 Holland SK, Hessler RB, Reid-Nicholson MD, Ramalingam P and Lee JR: Utilization of peripherin and S-100 immunohistochemistry in the diagnosis of Hirschsprung disease. Mod Pathol 23(9): 1173-1179, 2010. PMID: 20495540. DOI: 10.1038/modpathol.2010.104

12 Chi S, Fang M, Li K, Yang L and Tang ST: Diagnosis of Hirschsprung's disease by immunostaining rectal suction biopsies for calretinin, S100 protein and protein gene product 9.5. J Vis Exp 26(146), 2019. PMID: 31081806. DOI: 10.3781/ 58799

13 Christensen MV, Høgdall C, Jensen SG, Lokman N, Ricciardelli C, Christensen IJ, Christiansen P, Brask J, Karlsen MA, Nissen TK, Jochumsen KM and Høgdall E: Annexin A2 and S100A10 as candidate prognostic markers in epithelial ovarian cancer. Anticancer Res 39(5): 2475-2482, 2019. PMID: 31092442. DOI: 10.21873/anticanres. 13367
14 Liao X, Abu-Farsakh SH and Zhang D: Sarcomatoid renal cell carcinoma with unusual metastasis to the small intestine manifesting as perforated appendicitis. In Vivo 33(6): 22252228, 2019. PMID: 31662560. DOI: 10.21873/invivo.11726

15 Cimpean AM, Lalosevic D, Lalosevic V, Banovic P, Raica M and Mederle OA: Disodium cromolyn and anti-podoplanin antibodies strongly inhibit growth of BHK 21/C13-derived fibrosarcoma in a chick embryo chorioallantoic membrane model. In Vivo 32(4): 791-798, 2018. PMID: 29936460. DOI: 10.21873/invivo.11309

16 Meyrat BJ, Lesbros Y and Laurini RN: Assessment of the colon innervation with serial biopsies above the aganglionic zone before the pull-through procedure in Hirschsprung's disease. Pediatr Surg Int 17(2-3): 129-135, 2001. PMID: 11315271. DOI: $10.1007 / \mathrm{s} 003830000507$

17 Yoshimaru K, Taguchi T, Obata S, Takemoto J, Takahashi Y, Iwanaka T, Yanagi Y, Kuda M, Miyoshi K, Matsuura T, Kinoshita Y, Yoshioka T, Nakazawa A and Oda Y: Immunostaining for $\mathrm{Hu} \mathrm{C} / \mathrm{D}$ and CD56 is useful for a definitive histopathological diagnosis of congenital and acquired isolated hypoganglionosis. Virchows Arch 470(6): 679-685, 2017. PMID: 28424865. DOI: $10.1007 / \mathrm{s} 00428-017-2128-9$

18 Chisholm KM and Longacre TA: Utility of peripherin versus MAP-2 and calretinin in the evaluation of Hirschsprung disease. Appl Immunohistochem Mol Morphol 24(9): 627-632, 2016. PMID: 26469323. DOI: 10.1097/PAI.0000000000000241

Received January 16, 2020

Revised January 29, 2020

Accepted February 3, 2020 